\title{
Precise Measurement of Process Bias and its relation to MEEF
}

\author{
Terrence E. Zavecz - TEA Systems \\ tzavecz@TEAsystems.com \\ Phone: (+01) 6106824146
}

Copyright 2007, Society of Photo-Optical Instrumentation Engineers and TEA Systems Corp.

This paper was published in SPIE Vol 6520-139 and is made available as an electronic reprint or preprint with permission of SPIE. One print or electronic copy may be made for personal use only. Systematic or multiple reproduction, distribution to multiple locations via electronic or other means, duplication of any material in this paper for a fee or for commercial purposes or modification of the content of the paper are prohibited. 


\title{
Precise Measurement of Process Bias and its relation to MEEF
}

\author{
Terrence E. Zavecz \\ TEA Systems, 65 Schlossburg St., Alburtis, PA 18011 USA; tzavecz@TEAsystems.com
}

\begin{abstract}
Process Bias is traditionally defined as a manufactured offset of the mask-features that induces a photoresist image size to more closely match the nominal or desired circuit design size. The metric is calculated as the difference between the size of the image on the wafer and the mask with image reduction taken into consideration.

Optical process corrections (OPC) in the mask design must consider not only the Bias but also the influence of aerial image artifacts such as near-neighbor proximity, polarization and birefringence. The interactions are further complicated by the wavefront's interaction with the imaging media and optical interactions with the translucent film stack on the wafer. With the increased frequency of resolution enhancement (RET) artifacts on the mask, the concept of Bias as a simple scalar becomes less clear.

In this study Bias is shown to exhibit the anticipated systematic response to all of the static exposure conditions of the process. Variations across each field-of-exposure however behave nonlinearly with the range of fluctuations encountered within the process-space experienced during device manufacture. A model is developed that allows the Bias response to be comparatively measured for each mask feature-design that characterizes not only the behavior at optimum exposure but also each features stability across process and imaging perturbation sources..

The Bias models are applied to profile metrology gathered from matrix exposure data. Fine-structure perturbations in the Bias are extracted comparing their relative variation to process fluctuations that in-turn illustrates a strong individual feature construction-sensitivity. This analysis suggests that individual feature design is a strong contributor to processstability of a reticle. Even more significant, the Static Bias variation across the exposure field of a reticle is shown to be inversely related to the dose-uniformity map needed to achieve uniform critical features at the process-target size.

A new metric is introduced to provide a means of modeling the non-linear local Bias Signature for IntraField feature perturbations as a measure of the Bias Error Enhancement Function (BEEF). The BEEF metric is shown to be relatively insensitive to variations in the manufacturing exposure process-space but strongly responsive to variations in critical feature manufacture or design. The model is then extrapolated to define the relationship between Bias Response and the Mask Error Enhancement Function (MEEF).

The base design of a photomask feature is shown to be a strong contributor not only to resolution and depth-of-focus but also to the robustness of image response or it's ability to maintain stable resolution and depth of focus across the process-space. The Proper selection of different feature design alternatives can greatly reduce photomask sensitivity to process variations. The selection process for these designs as well as new reticle validation is simplified using the BEEF metric as an evaluator. "BEEF" is a metric more closely tied to process response of a reticle design than MEEF and is more easily extracted from in-process raw metrology.
\end{abstract}

Keywords: DFM, Bossung, Dose Control, ACLV, MEEF, BEEF, Bias, Process Space, Photomask, Dose Uniformity, Feature Design, Modeled Response, DoseMap, Bias Error Enhancement Function

\section{MOTIVATION AND INTRODUCTION}

\section{Removing the Mystery of MEEF}

Considering the business maturation of the semiconductor business, there is a surprising level of variation in the annual planning needed to maintain a course consistent with the long-term technical goals of the semiconductor industry. A long-view of the annual ITRS Roadmap illustrates the volatility and struggle underway to technically maintain a pace consistent with the market demands. The 2005 ITRS Roadmap exemplified this effort in an article in MICRO magazine that stated "...the expectation now is that different device parameters will scale at different rates...". . Driving this statement is the realization that low contrast, or 'Low k1' process design structures whose sizes approach the Rayleigh 
Limit are highly sensitive to the toolset and process specifics of each facility as well as the ultimate size of the structure in production. Features in this realm therefore replicate on the wafer at different rates than their local neighbors complicating manufacturing's efforts to maintain stable production as control variables drift within the process space.

One key industry metric used to monitor the process stability of device scaling that maintains a direct link to Across Chip Linewidth variation (ACLV) is the Mask Error Enhancement Factor (MEF or MEEF). The MEEF formula is a very pliant metric used to describe the response of the wafer image relative to variations in size of the reticle feature. More specifically, the MEEF metric describes the deviation from a constant reticle-wafer Bias for the process as a rate of change of the wafer image in the resist driven by small changes in the corresponding reticle feature. Image uniformity improves and critical feature size-excursions are reduced as MEEF values are reduced. Industry goals target MEEF values to be maintained at about 2.2 for photomask design performance of the smallest, most critical images over the next decade.

From a designer-simulation vantage, the concept of a specific single variable is most useful when trying to validate the range of a mask design to a generalized process requirement. Simulations that account for the reactive elements of the photoresist typically characterize the transition from the reticle-object to the final imaged profile in the photoresist by taking into account the photo-chemical interactions of the films with little regard to the aberrations specific to the exposure toolset.

In the actual first implementation of a new design or

\begin{tabular}{|c|c|c|c|}
\hline Year & $\mathbf{2 0 0 7}$ & $\mathbf{2 0 1 0}$ & $\mathbf{2 0 1 3}$ \\
\hline DRAM 1/2 Pitch & 65 & 45 & 32 \\
\hline $\begin{array}{c}\text { CD Uniformity } \\
\text { (MPU) }\end{array}$ & 6.6 & 4.7 & 3.3 \\
\hline MEEF Isolated & 1.6 & 2.2 & 2.2 \\
\hline MEEF Dense & 2.2 & 2.2 & 2.22 \\
\hline
\end{tabular}

Table 1: ITRS Roadmap CD Uniformity goals mask layer the lithographer receives this specific design in the codified format of a hard binary or enhanced reticle. Prior to first use, this hard-coded pattern must be validated for transfer under restrictions imposed by the device fabrication specification to determine if there is sufficient process range for yielding functional end product. Any lithographer soon discovers that individual photomasks of the same device layer respond differently and that each unique gate or via on the reticle can respond very differently from it's near neighbors of the same design. MEEF therefore does not provide the proper metric for pattern-specific performance characterization as applied to the reality of production variations and process mix.

In this spirit we address the needs of the sub-70 nanometer $(\mathrm{nm})$ device nodes by empirical modeling of the additional perturbations introduced during the convolution of the reticle image with the process. A direct measurement of the reticle-to-wafer Bias of critical features is investigated first under stable exposure -- focus and dose -- to deconvolve the relative contributions of full-wafer Bias variation. Next the test reticle is exposed in a focus and dose matrix whose bounds mimic those encountered in the normal process control-space or "process-space" of manufacturing.

A focus-exposure matrix experiment was setup and run over several "193" scanners. Data was measured using both CD-SEM and Scatterometry. As expected, Bias is shown to be highly systematic and stable across the lens and at the same time sensitive to exposure variations in focus and dose. Historic definitions of Process Bias treat the metric as an unchanging offset of the wafer image size from that of the reduced reticle feature. In practice, Bias variations are observed to be systematic and predictable across the process-space as well as the field of exposure. Drawing on the classic definition of the Mask Error Function, a new metric describing the response of a specific reticle feature designs local variation of wafer-Bias with small changes in the manufactured reticle-feature size is derived and characterized. This metric now provides a tool for the device designer as a more direct link to understanding reticle sensitivity to each user's process-space interactions.

\section{FIXED-FOCUS BIAS VARIATION}

Reticle feature sizes can be reliably measured by either classic CD-SEM or optical ellipsometric technologies. ${ }^{\text {ii }}$ Similarly, the characteristic signature of the reticle can be removed from the process-wafer measured data obtained from test or product reticles to accurately model the true response of process and toolset perturbations in the manufacturing sequence using commercially available software such as the Weir PW software from TEA Systems.

Wafer Bias is derived from this metrology as the difference between the wafer size of a feature and it's corresponding 
size on the reticle when standard demagnification of the process is taken into account:

$$
\text { Wafer Bias }=\mathbf{C D}_{\text {wafer }}-\mathrm{CD}_{\text {reticle }}=\mathrm{W}-\mathbf{R}
$$

Reticle feature-size variations are consistent and slowly changing across the field of the device object. The imaged final size of each reticle feature incorporates variations containing both a static signature, that is fixed and un-varying with exposure to the wafer, and dynamic component that is sensitive to process-space variations and tool aberrations. Dynamic signature levels are highly sensitive to the process stability and quality of the photomask manufacturer and are more commonly associated with photomasks employing the various Reticle Enhancement Techniques (RET). Static reticle perturbations will be the dominant contributor for pure binary reticles not employing enhancement techniques. ${ }^{\text {iii }}$

Static signature removal is a simple matter of subtraction when the features measured on the reticle correspond to the exact same features in each field measured on the wafer. Orientation and alignment to the proper structures is critical for mirrored and rotated data patterns.

When the wafer-measured patterns are not the same as those measured on the reticle or are a subset of the data then the process increases in complexity. The reticle data must be modeled and interpolated to determine the appropriate feature size at the points measured on the wafer-image. A "best-fit" is then applied to insure proper alignment and scaling of the full-field signatures of reticle and wafer. Commercial software packages, such as Weir ProMEEF, must be employed under these circumstances and this is the tool used in the analysis of the data presented in this discussion.

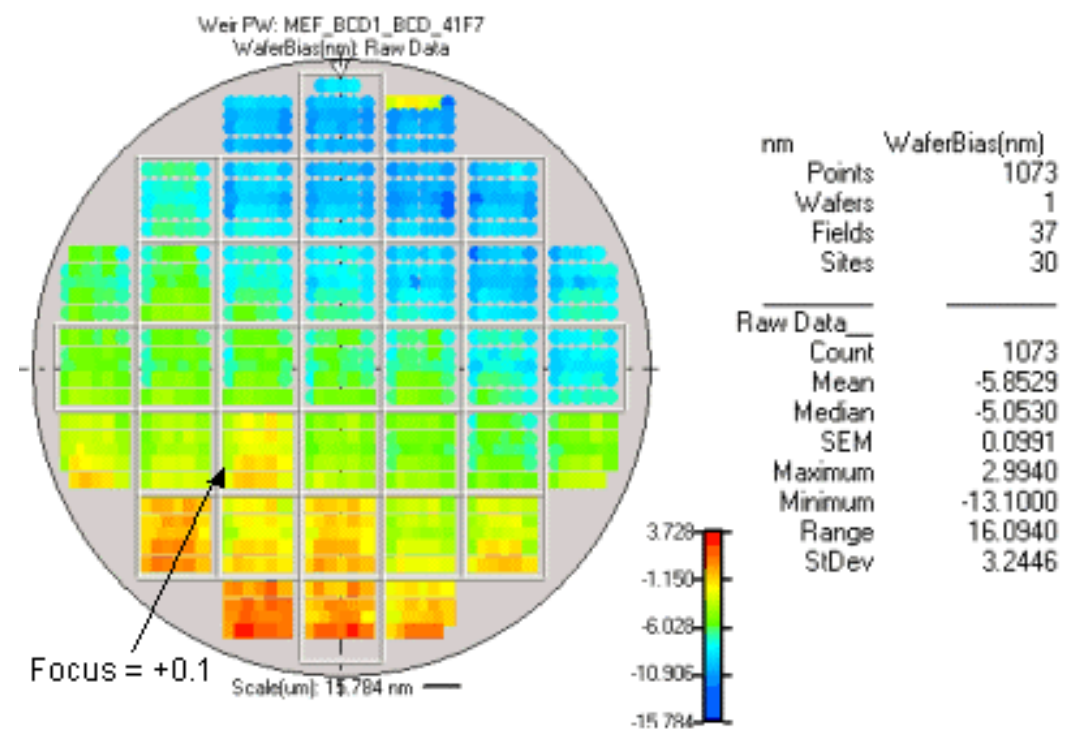

Figure 1: Fixed-exposure raw-data variation of reticle-to-wafer Bias

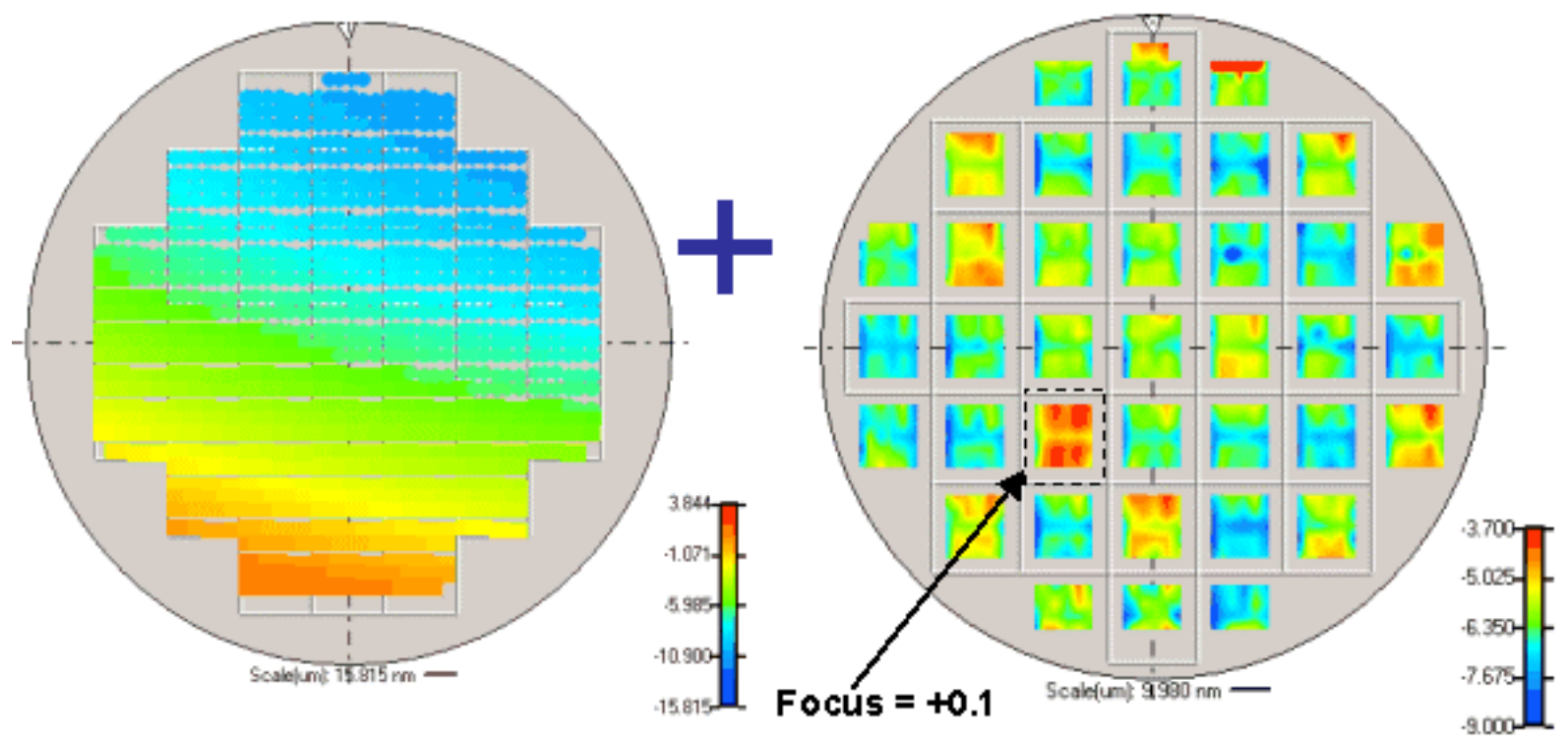

Figure 2: Wafer-Bias across a fixed-exposure wafer. Bias is modeled for the slowly changing process variation across the wafer (left) and the resulting IntraField details of each exposure (right). 


\section{Fixed-Exposure Analysis of Wafer Bias}

Figure 1 exhibits a full-wafer of $70 \mathrm{~nm}$ target-size half-pitch features as measured, using a Nanometrics 9030 OCD Tool and after the removal of the reticle signature using Weir ProMEEF software. The plot of figure 1 shows a distinct topto-bottom variation in Bias offset as a "tilt" or contour-slope. This tilt is clearer in the wafer-component model of figure 2.

The tilt in across-wafer feature Bias is process, not toolset or reticle derived. It contributes, in this instance, approximately $10 \mathrm{~nm}$ and is a known artifact of the Post Exposure Bake hot plate of this system. IntraField Bias variation in figure 1 appears as a faint but consistent variation of Bias across each field illustrating that the Bias of a single exposure is not constant across a single exposure. One field shown in the lower left quadrant of figure 1 was intentionally offset by defocusing the scanner by +0.01 micrometer (um) to assist in calibration of the analysis.

A Weir wafer-model is next applied to the data of figure 1 in order to extract the IntraField response of figure $2 .^{\text {iv }}$ IntraField Wafer Bias ranges $4.3 \mathrm{~nm}$ in figure 2 with a relatively constant IntraField Bias signature across each field. The focus-offset field exhibits approximately $4 \mathrm{~nm}$ of feature-size increase above the surrounding exposure sites. InterField variations appear across the remainder of the wafer exhibiting a relatively stable field signature with the primary differences due to normal field-to-field defocus variation of the scanner.

The left-side graphic of figure 3 was created by picking a random, single-field plot from the IntraField full-wafer graphic on the right side of figure 2. This plot provides a image typical of the stable Bias Variation Signature across the exposure field. In comparison, the right side of figure 3 is a plot of the Dose Uniformity of the same exposure tool previous published by the author. ${ }^{\mathrm{V}}$ In that publication Dose Uniformity was defined as the variation in IntraField Dose needed to achieve a constant target feature size. In deriving uniformity a focus-dose matrix of exposures was used and the local variations in feature size due to Intra-Field focus errors were removed. The DoseMap of the Dose Uniformity plot did not draw upon any reticle-metrology.

The Dose-Uniformity contour plot can now be seen to be a negative image of the Bias Uniformity of the exposure tool. The smaller gray-scale plot adjacent to the color plots assist in this recognition. The DoseMap, Dose Uniformity derivation methodology is thus validated by an IntraField Bias calculation and can be derived without a-priori knowledge of the reticle.

IntraField Bias is an artifact of both the process and the exposure tool lens perturbations. In this instance, we have seen that full-wafer process variations, such as those from a bake-plate, are the greater source of Bias variation. We have also observed that the true reticle-Bias can be accurately derived for a given exposure tool by estimating the Dose Uniformity across the field needed to achieve proper target feature size without the need for reticle measurements.

\section{FORMALIZATION OF WAFER-BIAS PROCESS SENSITIVITY}

In the previous section the Wafer Bias was shown to be sensitive to both process and exposure-tool variation. These variations are not random but are stable and systematic and can be mathematically characterized by an equation

BCD Bias Uniformity
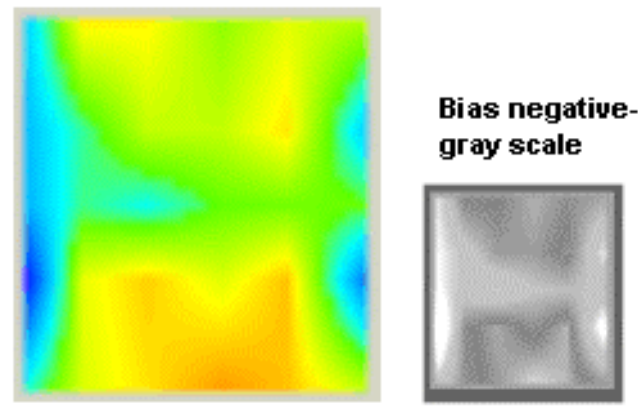

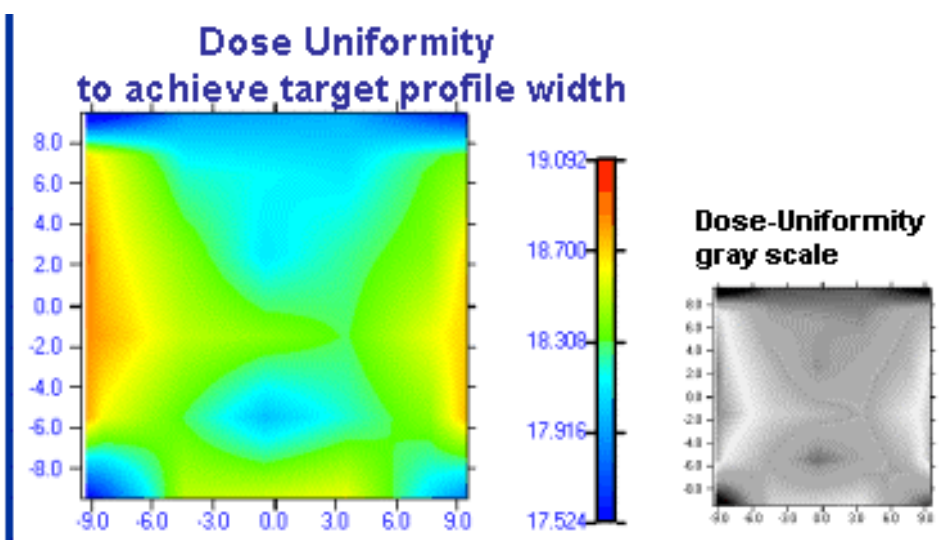

Figure 3: Link between IntraField Bias Uniformity (left) and the modeled Dose Uniformity (right) for the same exposure tool. The grey-scale images assist in recognition of the similarity in IntraField signature. 
construct as a product of a sensitivity coefficient " $\Lambda$ " that replicates the variables response to a process disturbance " $m$ " in a function of the form:

$$
F R_{a}=\Lambda_{a} * D_{m}
$$

Where the feature response (FRa) in this application is the Wafer Bias and the process disturbances "Dm" will be the slowly varying but stable Bias signature produced across the exposure field aberrations induced by the exposure tool. A Process Response Model that does not favor the slit and scan exposure strategy of the scanner can be constructed as:

$$
F R_{a}(r, \theta)=\beta_{o}+\sum_{j=1}^{n} \beta_{j} * r^{j} *(\cos (j \theta) * \sin (j \theta))+R_{a}
$$

FRa represents the feature response of the Wafer Bias over a single field of exposure such as shown in figure 3. The offset or $\boldsymbol{\beta}$ o coefficient of equation 3 describes the reticle-to-wafer Bias experienced by the field. Bias non-uniformity across the field that is due to illumination, effective exposure and lens aberrations will be incorporated in the remainder of the coefficients of the expansion. Rapid changes in wafer Bias due to local noise, measurement error or damaged individual-feature design will be found in the residuals Ra.

\section{Setup of the Experiment}

A focus-dose, or "FEM" matrix using the reticle employed in figure 1 was created to replicate the range of focus and dose excursions experienced as part of the exposure sequence of the process-space. The substrate was exposed by varying exposures from +0.24 um to -0.24 um defocus and exposure-dose from 18 to $24 \mathrm{mj} / \mathrm{cm} 2$. Grating consisting of $70 \mathrm{~nm}$ half-pitch features were exposed in a $193 \mathrm{~nm}$ scanner and thirty sites-per-field measured using a Nanometrics 9030 Optical CD tool. Weir ProMEEF, from TEA Systems, was then used to remove the measured-reticle from the measured Bottom Feature data (BCD). The resulting file containing the calculated BCD Bias data was loaded into Weir PW for additional analysis and modeling.

\section{Feature Bias Process Sensitivity}

The results of an analysis of the full-field Bias response of the reticle and its relation to exposure are presented in figure 4. Each data point represents the modeled Bias-offset of the exposure. The "Error Bars" associated with the points represent the systematic variation of Bias as it ranges across the modeled field of exposure.

Bias exhibits the following characteristics:

1. As is found in normal CD response curves, The Wafer-Bias data exhibits a $2^{\text {nd }}$ order, base response to focus. The variation about the best-fit curve however is significantly more stable and precise than is found in the normal process-window "Smile" analysis.

2. IsoFocal response is achieved for this pattern at $21 \mathrm{mj} / \mathrm{cm} 2$ dose with a Bias offset of about $28 \mathrm{~nm}$.

3. Increasing dose reduces the feature size until 23 $\mathrm{mj} / \mathrm{cm} 2$. Above this dose Bias reverses direction with the $24 \mathrm{mj} / \mathrm{cm} 2$ value exhibiting an equal Bias offset but with reduced focus range.

4. Full-field variation of Bias reduces in value as dose increases and then maintains a minimum at the optimum focus.

As anticipated, full-field BCD

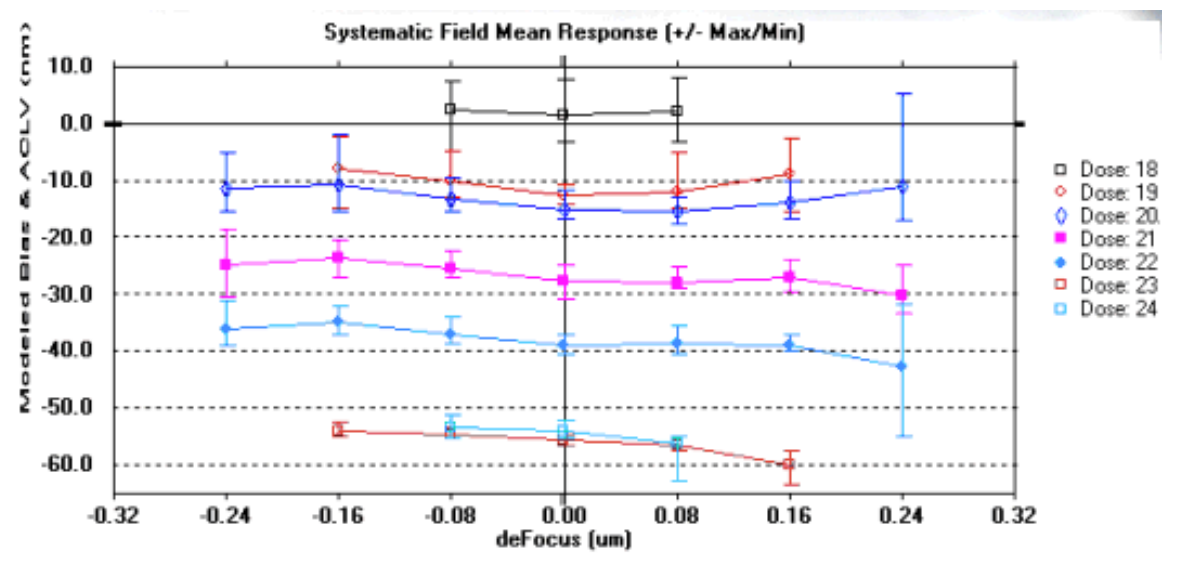

Figure 4: Full-Field modeled reticle bias response to defocus and dose. 
feature Bias is shown to be sensitive to focus in figure 4. A closer study of the response can be shown in figure 5 as a plot of the full-field across chip linewidth variation (ACLV) of Bias as a function of focus and dose. Figure 5 demonstrates that proper selection of both dose and focus for the exposure results in more than a constant offset in feature size. The optimum dose of $21 \mathrm{mj} / \mathrm{cm} 2$, corresponding to the IsoFocal dose, results in the greatest depth of focus for the feature as well as the lowest variation in ACLV over the range of defocus.

From the range of ACLV plotted in the figure, we can see that feature size stability improves consistently as the exposure-dose increases. The depth of focus stabilizes with $20 \mathrm{mj} / \mathrm{cm} 2$ and this extends to $22 \mathrm{mj} / \mathrm{cm} 2$. Even greater improvement in ACLV

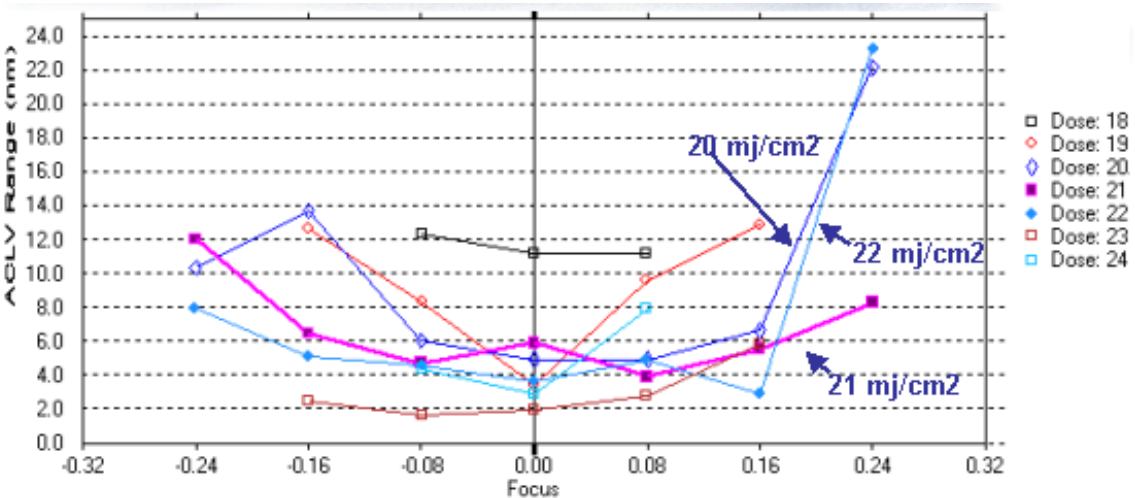

Figure 5: Feature-Bias across chip feature variation (ACLV) with exposure. compared to the $21 \mathrm{mj} / \mathrm{cm} 2$ value can be achieved by overexposing to 23 or $24 \mathrm{mj} / \mathrm{cm} 2$ but, while the feature image variation stabilizes with these exposures, the exposure also results in a reduction in the depth of focus.

A variation of Bias with dose, shown in figure 6, does not exhibit a pure, linear response. This is primarily due to the non-uniformity of focus across the aerial image of the scanner. In this figure, the ACLV variation, shown by the error bars of each point, appears larger for the IsoFocal 21 $\mathrm{mj} / \mathrm{cm} 2$ dosage. This is due to the increased range of defocus present in the data. If featuresize differences due to focuserrors are removed from the data of this graph then the response curve of figure 6 increases in linearity and a doseuniformity plot as shown in the right side of figure 3 can be created.

The nonlinear response shown in this graphic is augmented by the response variation of the

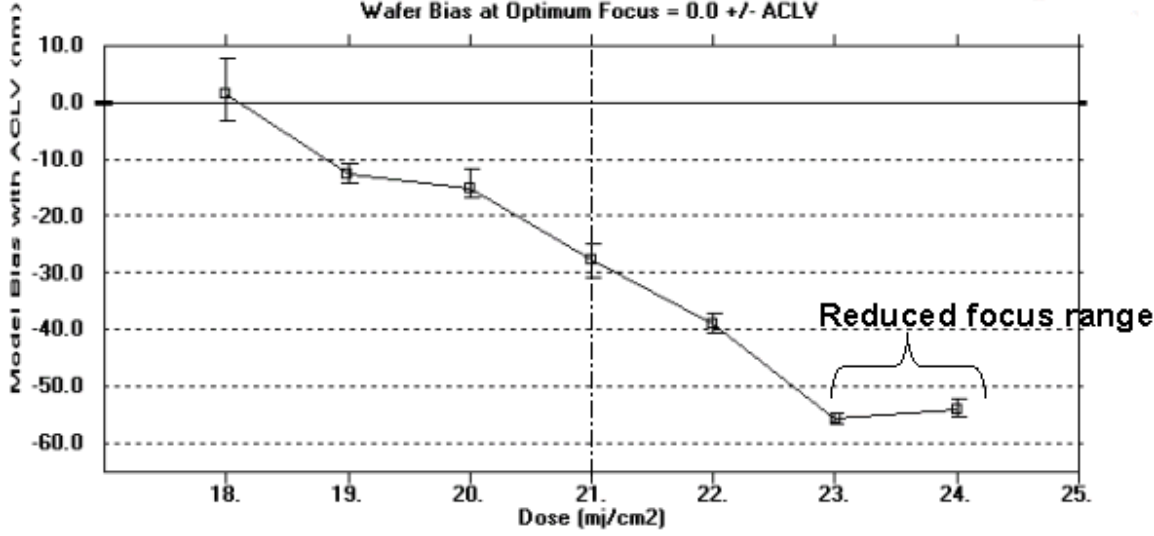

Figure 6: Feature-Bias response to variations in exposure-dose.

full-field data and suggests a distortion sensitivity of feature Bias. It is apparent that a photomask feature's linear Bias response cannot be assumed but must be controlled by improving the robustness of a feature's design to process-space variations.

\section{Non-linear Bias Response}

Drawing on past work in similar analysis endeavors we can parallel Exposure-Bias response nonlinearity to that of the Mask Error Enhancement Function of the "MEEF" variable. MEEF is defined as the variation in feature size on the wafer given a change in the demagnified reticle size feature or:

$$
M E E F=m * \frac{\partial C D_{\text {wafer }}}{\partial C D_{\text {Re ticle }}}=\frac{\partial W}{\partial R}
$$

Where " $\mathrm{m}$ " is defined as the magnification of the exposure tool, which is $4 \mathrm{x}$ in today's scanners. The MEEF variable for a well-behaved process will be equal to 1.0 signifying an equal change in wafer-size given an incremental change in 
reticle feature. ITRS MEEF criteria restrict this factor to target values of MEEF $<=2$ for low-contrast processes.

Experimentally derived MEEF can be extracted from a regression of the reticle and wafer measured features using the construct: ${ }^{\mathrm{vi}}$

$$
W=W o+\frac{\partial W}{\partial R} * R=W o+M E E F * R
$$

The Mask Error Enhancement Function or MEEF is annually reported as a mask-maker's problem in the ITRS roadmaps. However the function is not stable with the photomask signature and it's volatility across the exposure field and process-space has been shown to be highly sensitive to both exposure tool aberrations and the interaction of the feature design with these aberrations. ${ }^{\text {vii }}$

Drawing on the definition of Bias in equation 1, we next present a format for nonlinear Bias by paralleling the construct of equation 5 with:

$$
\text { Bias }=B o+\frac{\partial(W-R)}{\partial R} * R
$$

Where the nonlinearity of the Bias Response is here modeled as a change in the Wafer Bias (W-R) that results from a corresponding local change in the reticle size. Similar to the MEEF construct we define this variable as the Bias Error Enhancement Function or "BEEF" of the process and equation 6 can then be presented as:

$$
\text { Bias }=B o+B E E F * R
$$

Similar to MEEF, the BEEF variable is dimensionless in the classic nomenclature typified by " $\mathrm{nm} / \mathrm{nm}$ ".

Weir ProMEEF software calculated the local rate-of-change in the Bias or BEEF for each reticle site in every exposure field using the $70 \mathrm{~nm}$, Focus-exposure matrix (FEM) data presented previously. The resulting BEEF uniformity with dose variation across the dose range at the optimum focus is shown in figure 7.

The BEEF values are calculated to reside in the 0 to 1 range with the exception of the strongly underexposed $18 \mathrm{mj}$

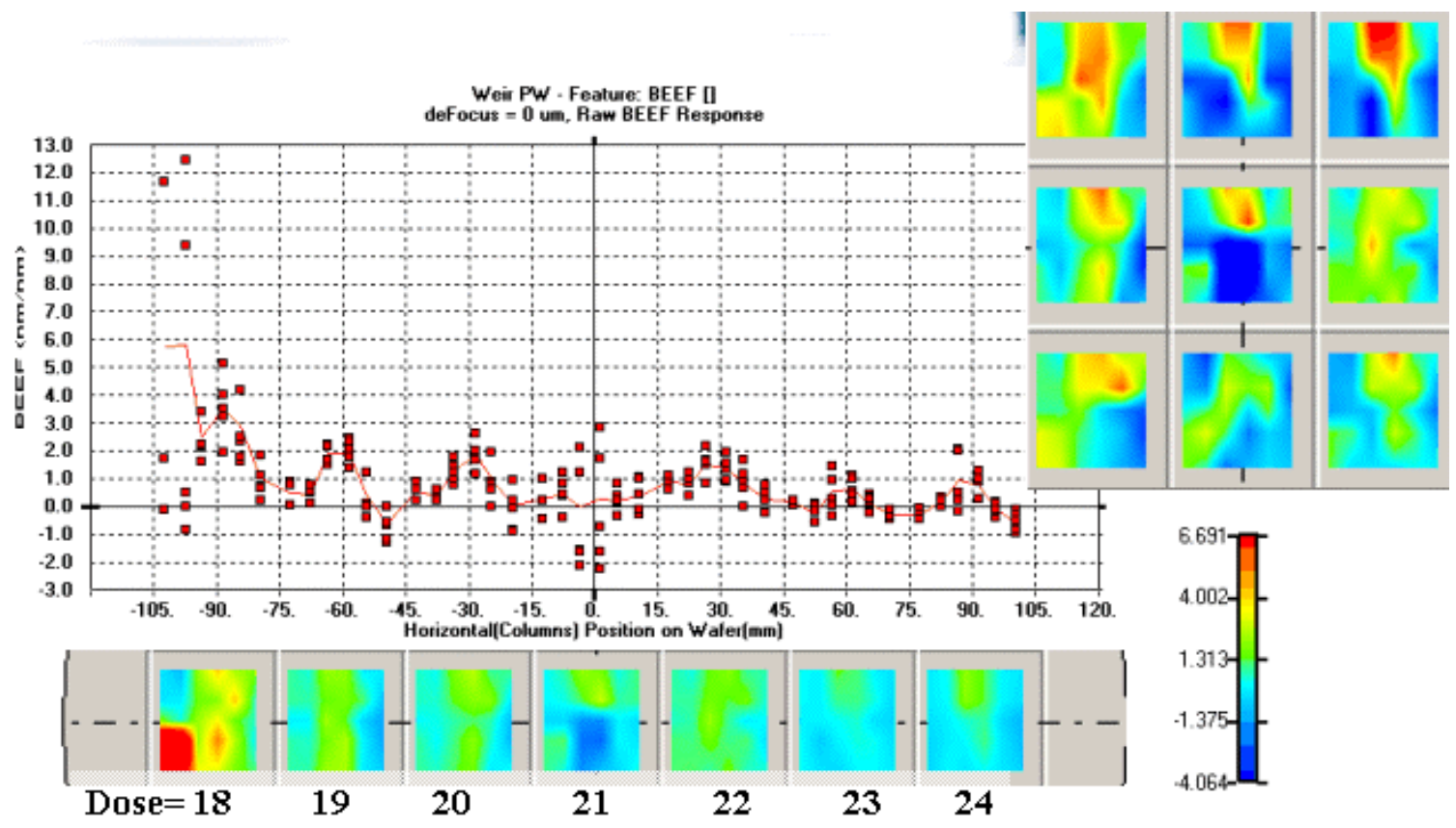

Figure 7: BEEF; nonlinearity of Bias with dose at 0 deFocus; 70nm, 1:1. Upper Right; Local BEEF signatures across 3x3 FEM matrix of wafer center. 

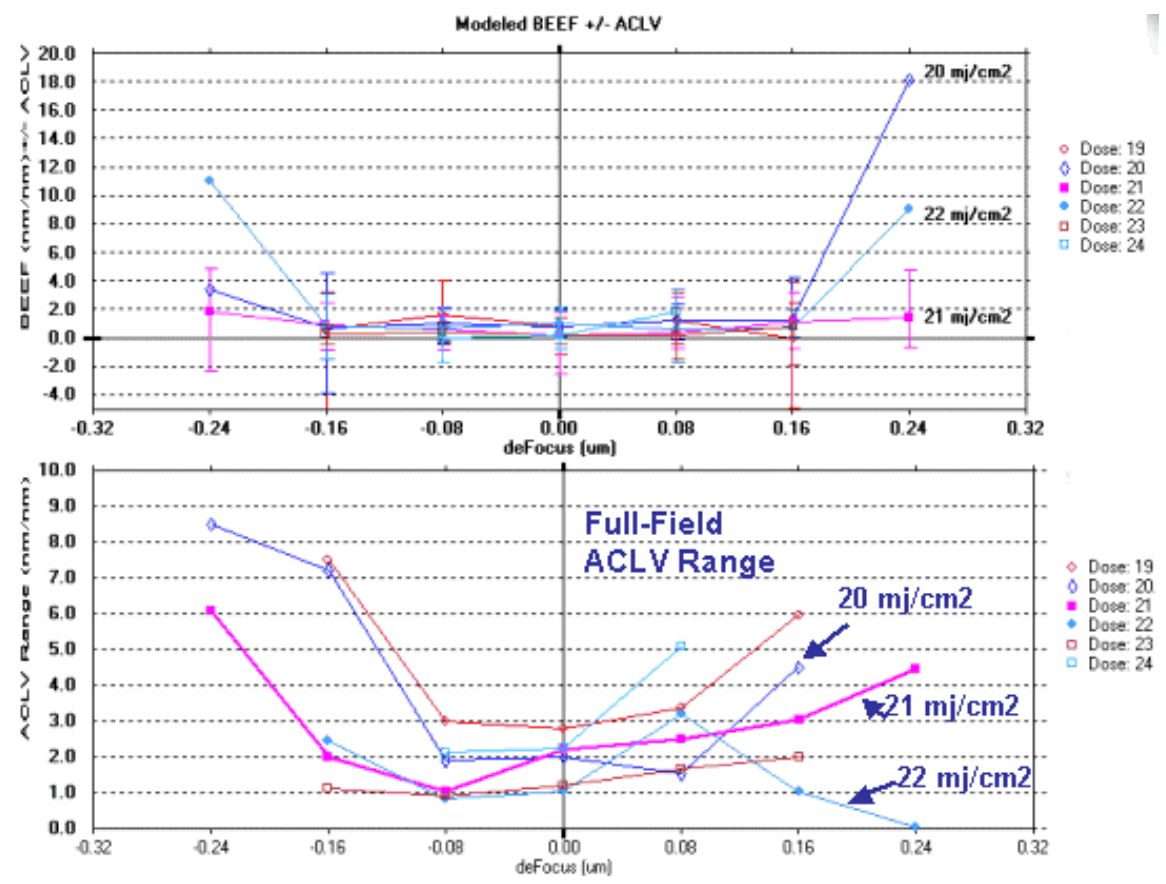

Figure 8: BEEF Process Response Sensitivity.

Top: BEEF response +/- ACLV variation with focus and dose Bottom: ACLV range of BEEF.

field. BEEF is relatively stable with respect to exposure across the field except for the optimum dose/ defocus field at the graph center where the BEEF unexpectedly expands across the exposure. The reason for this expansion is unknown at this time however it implies that the optimum exposure for a reticle exhibits the greatest sensitivity to reticle size change.

We've now shown that the BEEF signature is highly sensitive to exposure conditions. We can further examine it's response using an expansion from equation 6 as:

$$
B=B o+\left[\frac{\partial W}{\partial R}-\frac{\partial R}{\partial R}\right] * R
$$

or, in more compact terms, derived by the implementation of equation 1 :

\section{$B=B o+(M E E F-1) * R$}

Equation 9 therefore illustrates the relationship between BEEF and MEEF. BEEF is directly related to the MEEF of the reticle and the MEEF itself now has a direct link to the feature design response on the mask. This relationship implies that the BEEF function is highly sensitive to the convolution of the aberrations of the exposure tool, the process film response and the designed sensitivity of the feature itself. This designsensitivity is treated in greater detail in a paper recently presented by the author. ${ }^{\text {viii }}$

Next the full-field response of the reticle BEEF is modeled with Weir PW using the model of

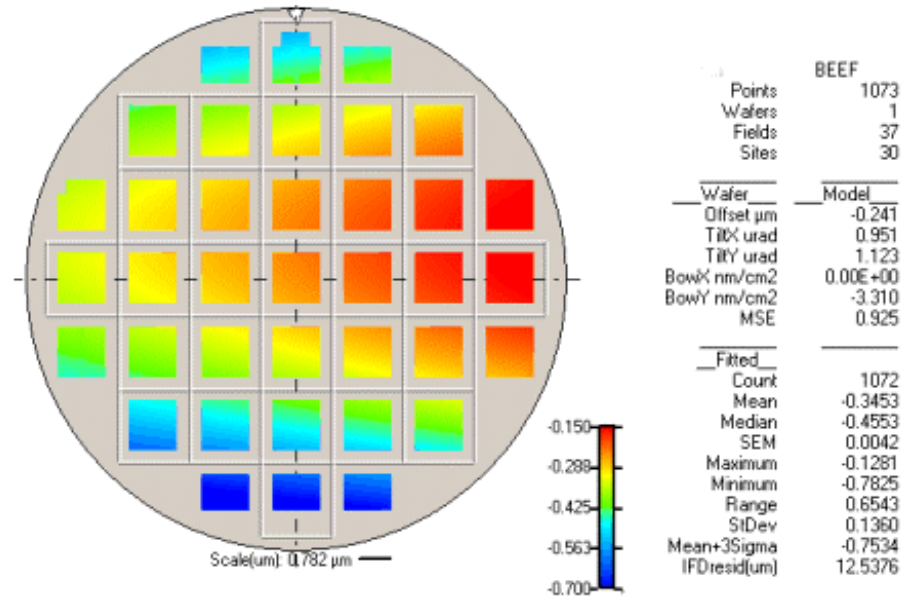

Figure 9: Modeled Process-Space response of BEEF 
equation 3. Results for BEEF sensitivity are presented in the top graph of figure 8. The BEEF metric appears to be even less sensitive to focus variation than previously seen for BIAS.

The BEEF metric is relatively insensitive to dose over the normal process space however there is a strong relation of the variable's ACLV as the dose is varied from the IsoFocal value. This ACLV response is clearly plotted in the lower graph of figure 8 where the across-chip response of BEEF exhibits focus sensitivity similar to the overall Bias.

A clearer vision of the process-space response of the BEEF metric for this reticle can be found in the wafer-signature contour plot of the modeled BEEF variation across the FEM substrate in figure 9. The process-space focus and dose sensitivity of the BEEF maximizes at a value equal to $-0.128 \mathrm{~nm} / \mathrm{nm}$ and its stability can be seen in the red-coded contour areas with a total process-contributed range of only $0.654 \mathrm{~nm} / \mathrm{nm}$.

This process in-sensitive behavior suggests that the source of ACLV Bias uniformity is a strong nonlinear response of the reticle or design construct rather than the process setup and variation.

\section{BEEF Evaluation of Reticle Feature Design Robustness}

The influence of feature design on BEEF, and therefore MEEF response can be seen in figure 10. This contour represents a BEEF derivation from a different reticle than the one of figure 7 that contains some known manufacture errors. Examinations of the FEM wafer contour plot for raw BEEF plotted in figure 10 clearly shows the relative process-space insensitivity of the BEEF metric. BEEF is varying $+/-1.8 \mathrm{~nm} / \mathrm{nm}$ primarily across the reticle field and independent of exposure.

Two areas on each reticle-exposure appear consistent as low and high BEEF points, changing contour intensity only as their interactions with neighboring sites change with the base exposure changes of each field.

Figure 11 illustrates this site-design dependency quite clearly. Site 18 yields the low BEEF values seen on the whole-wafer contour plot and site 21 yields a consistently high BEEF response. The BEEF variable is thus a consistent and easy metric for the evaluation of feature design robustness of response to variations in the manufacturing process-space. Similar to the conclusions drawn in reference \#7 previously cited, the individual feature design is the greatest contributor to process response and a feature's robustness to exposure variations across the process-space.

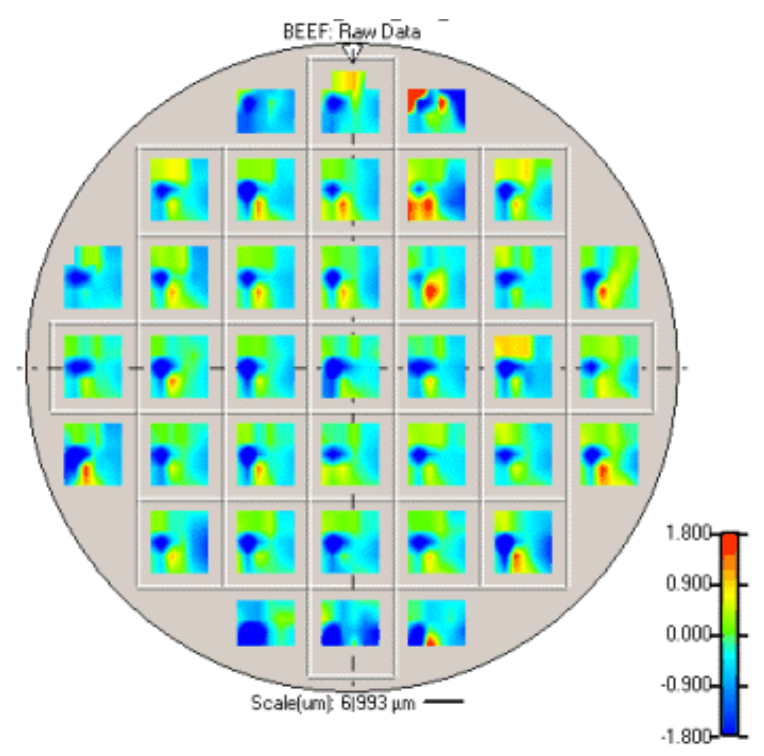

Figure 10: Raw BEEF variation across the FEM wafer matrix

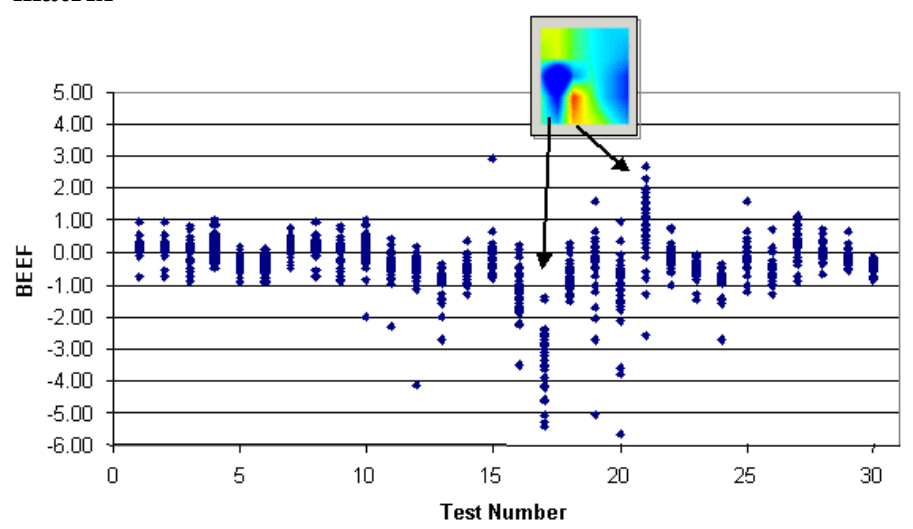

Figure 11: Raw BEEF variation with reticle site design. Contour plot is a representative exposure from figure10. 


\section{CONCLUSIONS}

- The Wafer Bias measured as across chip variation is minimized across an exposure at the IsoFocal dose.

- Wafer Bias and the required Dose Uniformity of a reticle to achieve target size are directly related and inverse in their response.

- Wafer Bias is not constant with exposure but varies across the field as a function of:

- Local aberrations,

- Robustness of the photomask feature design to process fluctuaions and

- Individual feature construction quality on the reticle during manufacturing.

- The nonlinear response component of Bias is called the Bias Error Enhancement Function and:

- Is relatively insensitive to exposure variations of focus and dose.

- Can be calculated from empirical Bias data as:

- $\quad \mathrm{Bias}=\mathrm{Bo}+(\mathrm{BEEF} *$ Reticle $)$ or

- $\quad$ Bias $=$ MEEF -1

- "BEEF" is a metric more closely tied to process response of a reticle design than MEEF and is more easily extracted from in-process raw metrology.

\section{REFERENCES}

i. MICRO magazine, Jan 2006, “Technology node’ retired as feature-scaling gauge”, pp. 12-17

2. ii. T. Zavecz, "Full-Field Exposure Control Implications of the Mask Error Function", SPIE (2006) 615512.

3. iii T. Zavecz, "Full sub-65 nm data-modeling for Photomask Manufacturing", FujiFilm Interface 2006

4. $\quad$ iv . M Dusa, R Moerman, B Singh, P Friedbergc, R Hoobler, T Zavecz, , "Intra-wafer CDU characterization to determine process and focus contributions based on Scatterometry Metrology", SPIE (2005) vol. 5378_11.

5. $\quad$ v. T. Zavecz, "Bossung Curves; an old technique with a new twist for sub-90 nm nodes", SPIE (2006) vol. $6152-109$

6. $\quad{ }^{\text {vi } . ~ I . ~ J e k u a c, ~ B . R o b e r t s, ~ C . H a m p e, " M a s k ~ E r r o r ~ F a c t o r ~-~ c r i t i c a l ~ d i m e n s i o n ~ v a r i a t i o n ~ a c r o s s ~ d i f f e r e n t ~ t o o l s, ~}$ features and exposure conditions", SPIE 4346-85 (2001)

7. vii . T. Zavecz, "Full-Field Exposure Control Implications of the Mask Error Function", SPIE 6155-12 (2005)

8. viii . T. Zavecz, "Methods for comparative extraction of OPC response", SPIE (2007) 6520-64.

9. 